\title{
Morphology of the interfaces between transition metals and their native oxides: Role of interdiffusion processes
}

\author{
Sebastien Couet, ${ }^{1}$ Kai Schlage, ${ }^{1}$ Karel Saksl, ${ }^{2}$ and Ralf Röhlsberger ${ }^{1, *}$ \\ ${ }^{1}$ Deutsches Elektronen Synchrotron (DESY), Notkestraße 85, 22603 Hamburg, Germany \\ ${ }^{2}$ Institute of Materials Research, Slovak Academy of Sciences, Watsonova 47, 04353 Kosice, Slovak Republic
}

(Received 27 November 2008; published 17 February 2009)

\begin{abstract}
The magnetic coupling phenomena appearing between a ferromagnetic transition metal and its antiferromagnetic oxide crucially depend on the composition and morphology of the interface. We present combined in-situ x-ray absorption spectroscopy and ex-situ x-ray reflectometry studies of the growth of a native-oxide layer and the interface formation with its metal parent. Two model systems, $\mathrm{Fe}$ and $\mathrm{Ni}$, are investigated. It is found in the case of $\mathrm{Fe}$ that oxidation and reduction processes appear upon deposition of metal on the oxygen-rich oxide. This results in symmetric metal/oxide and oxide/metal interfaces. Comparison with the case of $\mathrm{Ni}$, where no reduction is observed, shows that this symmetry is intrinsically linked to the underlying reduction process. These results have important consequences for the growth of transition-metal/oxide interfaces in general.
\end{abstract}

DOI: 10.1103/PhysRevB.79.085417

PACS number(s): 61.05.cj, 61.05.cm, 68.35.Ct

\section{INTRODUCTION}

In recent years, multilayers incorporating transition metal (TM) such as $\mathrm{Fe}, \mathrm{Ni}$, or $\mathrm{Co}$ and transition-metal oxide (TMO) layers have seen an increasing interest due to their potential use as building blocks in magnetic structures such as spin valves and magnetic tunnel junctions. ${ }^{1-3}$ In particular, some transition-metal monoxides $(\mathrm{FeO}, \mathrm{NiO}, \mathrm{CoO})$ possess an antiferromagnetic (AFM) state and are often used as pinning layers in exchange biased systems. ${ }^{4,5}$ Because exchange bias is intrinsically an interface effect, the morphology and structure of the ferromagnet/antiferromagnet interface plays a determining role in the observed magnetic properties., ${ }^{5,6}$ The growth of TM/TMO layer systems also presents some technical challenges. Often, the growth of TMO layers is performed by deposition of the metal in the presence of oxygen. ${ }^{3,7}$ This usually results in a direct growth of an oxide layer, but also in a partial oxidation of the underlying metal substrate. Regan et $a l^{8}{ }^{8}$ showed that oxidation and reduction processes at $\mathrm{TM}_{1} / \mathrm{TM}_{2} \mathrm{O}$ interfaces (where subscripts 1 and 2 refer to different transition metals) lead to relatively complicated interface structures.

Another approach is to take advantage of the oxidation properties of transition metals to grow oxide layers. A TM/ TMO interface (in the following, the nomenclature TM/TMO denotes a layer structure with the TM deposited first) can be created simply by controlled oxidation of a thin metal layer and subsequent deposition of the same metal. ${ }^{9,10}$ The advantage lies in the relatively good control of the oxide layer thickness and the easiness of preparation. However, it is difficult to control a priori the oxide stoichiometry and the degree of crystallinity. Several studies have been carried out on transition-metal/native-oxide systems (where nanometer thick oxide layers are produced by controlled oxidation at room temperature), showing that coupling effects occur between the magnetic oxide and the magnetic metal. ${ }^{9,11}$ In particular the discovery of noncollinear magnetic coupling of $\mathrm{Fe}$ layers separated by native-oxide spacers demonstrated that strong exchange coupling can arise at the interface between the metallic FM and the native-oxide AFM. A recent $\mathrm{x}$-ray absorption spectroscopy experiment ${ }^{12}$ showed that the native oxide embedded between two $\mathrm{Fe}$ layers adopts a structure similar to $\mathrm{FeO}$. It was shown that the creation of the $\mathrm{FeO}$ layer was due to strong interdiffusion processes occurring upon deposition of Fe metal on top of the oxygen-rich native oxide.

In this paper, we present combined in-situ hard x-ray absorption spectroscopy and $e x$-situ x-ray reflectometry studies of the interface formation between a native oxide and its metal parent. Two systems are investigated, namely, Fe and $\mathrm{Ni}$, to compare how the different growth processes influence the interface morphology. The x-ray absorption part gives a quantitative view of the interface chemistry during the oxidation and deposition process. The resulting interface morphology is then studied by $\mathrm{x}$-ray reflectivity experiments on multilayered samples. The different interface profiles obtained are discussed and related to the underlying oxidation and reduction processes arising during the growth. These findings have consequences for the appearance of long-range interlayer coupling in transition-metal/native-oxide systems in general.

\section{X-RAY ABSORPTION SPECTROSCOPY}

The in-situ $\mathrm{x}$-ray absorption experiments were carried out in a dedicated ultrahigh-vacuum deposition system ${ }^{13}$ mounted on the A1 beamline of the DORIS III storage ring (Hamburg, Germany). The base pressure was 1 $\times 10^{-9}$ mbar. Polycrystalline $\mathrm{Fe}$ and Ni layers were deposited by dc magnetron sputtering (Ar gas pressure 5 $\times 10^{-3}$ mbar) on superpolished $\mathrm{Si}(001)$ substrates. A $6 \mathrm{~nm}$ thick Pd buffer layer was deposited in situ to avoid unwanted oxidation at the $\mathrm{SiO}_{2} /$ transition-metal interface. Thin $\mathrm{Fe}$ and Ni layers (thickness ranging from 0.4 to $2 \mathrm{~nm}$ ) were stepwise oxidized at an oxygen partial pressure of $1 \times 10^{-5}$ mbar and subsequently covered with the same metal. At each step, absorption spectra were recorded at the $\mathrm{Fe} K$ edge $(7.1 \mathrm{keV})$ and the Ni $K$ edge $(8.3 \mathrm{keV})$ for the $\mathrm{Fe}$ and Ni layers, respec- 

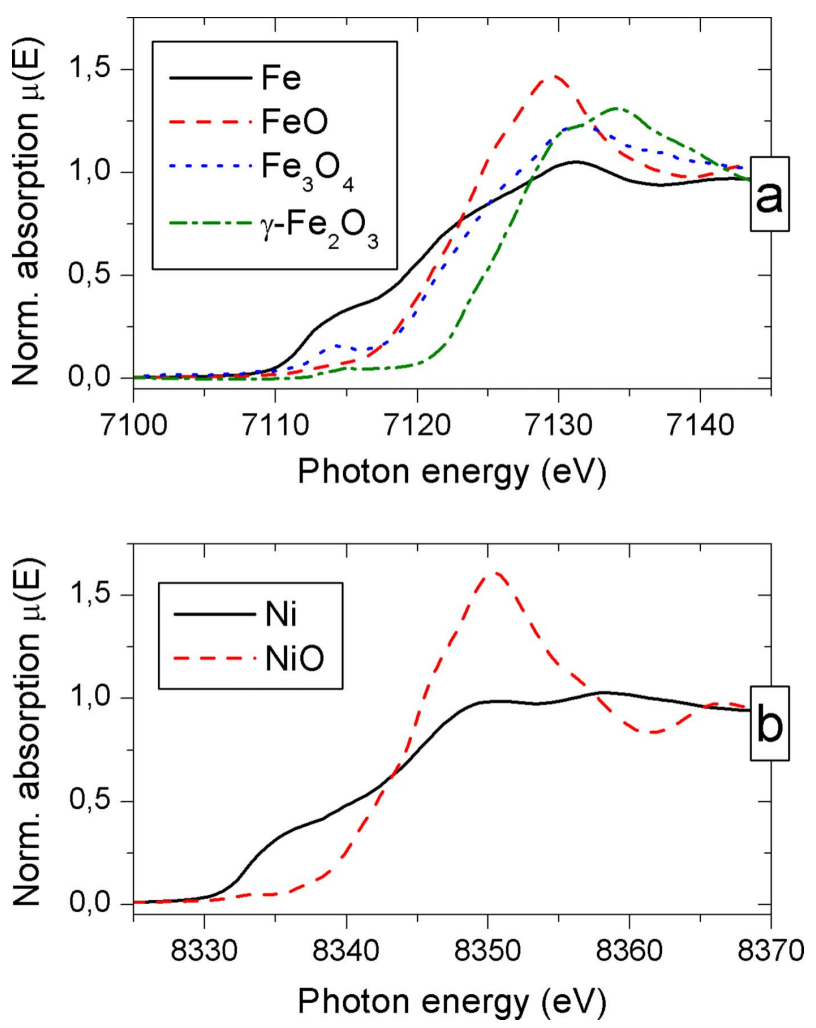

FIG. 1. (Color online) XANES $K$-edge spectra of reference samples for (a) $\mathrm{Fe}, \mathrm{FeO}, \mathrm{Fe}_{3} \mathrm{O}_{4}$, and $\gamma-\mathrm{Fe}_{2} \mathrm{O}_{3}$ and (b) $\mathrm{Ni}$ and $\mathrm{NiO}$.

tively. The absorption signal was recorded using a total electron yield detection scheme, which provides excellent signalto-noise ratio and sufficient probing depth (estimated to be around $50-100 \mathrm{~nm}$ for excitation by hard $\mathrm{x}$ rays ${ }^{14}$ ) to ensure that the whole layer system is probed. The absorption spectra were recorded up to $800 \mathrm{eV}\left(14.4 \AA^{-1}\right)$ above the absorption edge to cover the extended $x$-ray absorption fine structure (EXAFS) of the edge. Each $x$-ray absorption fine structure (XAFS) spectrum was recorded in approximately $30 \mathrm{~min}$. It was checked by repeated XAFS scans on a freshly deposited metal layer that the residual gas pressure does not influence the oxidation state of the layer over a few hours.

To obtain a quantitative measure of the composition of the layers, we analyzed the x-ray absorption near-edge structure (XANES) region of the absorption spectra. In the case that multiple phases are present in the sample under investigation, the XANES signal can be described as a linear combination of the XANES signals of the atoms in the different coordination geometries (sites). A quantitative measure of the composition of the layers was obtained by finding the best linear combination of the XANES spectra of reference oxide phases that fits the experimental spectra. From these results, the concentration of the different oxidation states $\left(\mathrm{Fe}^{0}, \mathrm{Fe}^{2+}, \mathrm{Fe}^{3+}, \mathrm{Ni}^{0}\right.$, and $\left.\mathrm{Ni}^{2+}\right)$ can be extracted. The oxide phases of transition metals can usually be described as a closed packed oxygen lattice into which metal atoms are placed in interstitial positions. ${ }^{15}$ Three different Fe sites can be distinguished in oxide phases: $\mathrm{Fe}_{\text {oct }}^{2+}\left(\mathrm{FeO}, \mathrm{Fe}_{3} \mathrm{O}_{4}\right), \mathrm{Fe}_{\text {tet }}^{3+}$ $\left(\gamma-\mathrm{Fe}_{2} \mathrm{O}_{3}\right)$, and $\mathrm{Fe}_{\text {oct }}^{3+}\left(\mathrm{Fe}_{3} \mathrm{O}_{4}, \gamma-\mathrm{Fe}_{2} \mathrm{O}_{3}\right.$, and $\left.\alpha-\mathrm{Fe}_{2} \mathrm{O}_{3}\right)$. The subscripts tet and oct refer to $\mathrm{Fe}$ in tetrahedral or octahedral
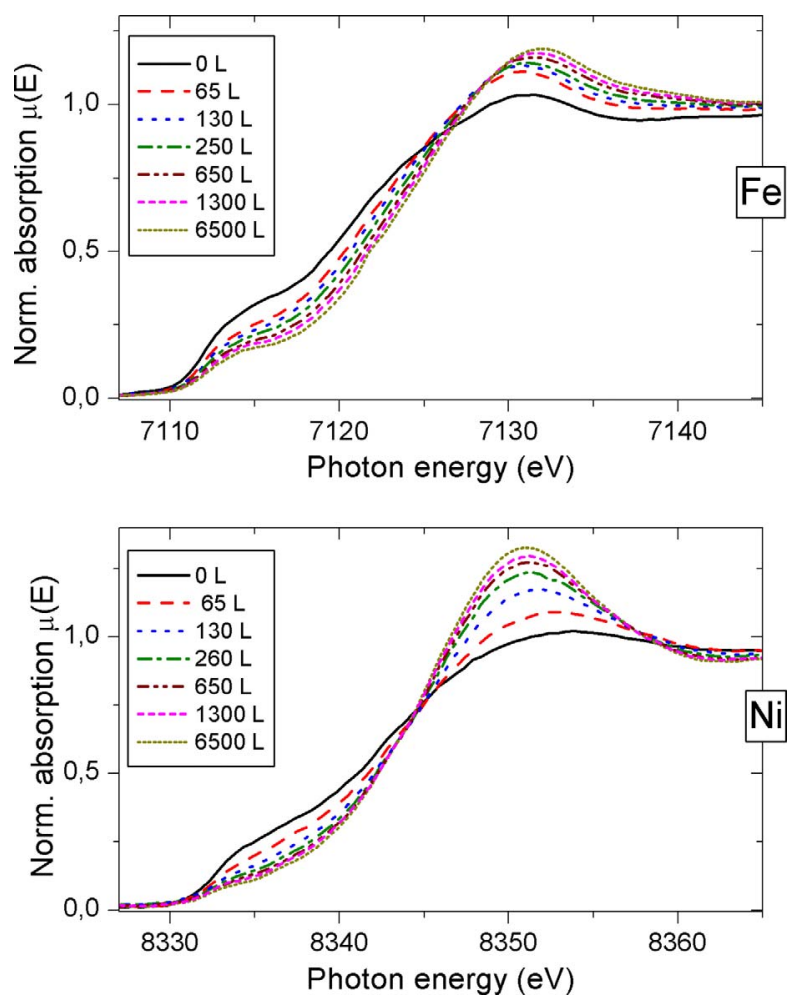

FIG. 2. (Color online) XANES $K$-edge spectra recorded at selected steps of the oxidation of (upper panel) a $1.2 \mathrm{~nm}$ thick Fe layer and (lower panel) a $1.4 \mathrm{~nm}$ thick Ni layer. The progressive shift of the absorption edge toward higher energies indicates an increase in the oxidation state of the sample with increasing oxygen exposure.

sites, respectively. For $\mathrm{Ni}$, the most common state is $\mathrm{Ni}_{\text {oct }}^{2+}$ (NiO). Reference spectra of the different oxide phases have to be measured in order to quantitatively determine the concentration of sites. For Fe, references for metallic $\mathrm{Fe}$ (a 50 $\mathrm{nm}$ thick layer deposited in situ), $\mathrm{FeO}$ (single crystal), $\mathrm{Fe}_{3} \mathrm{O}_{4}$ (powder diluted in a polyethylene matrix), and $\gamma$ - $\mathrm{Fe}_{2} \mathrm{O}_{3}$ (powder diluted in a polyethylene matrix) were measured. For Ni, reference spectra of metallic Ni (a $50 \mathrm{~nm}$ thick $\mathrm{Ni}$ layer deposited in situ) and $\mathrm{NiO}$ (powder sample) were used. The XANES spectra of the reference samples are shown in Fig. 1. Each reference spectrum has prominent features (onset of the edge, edge maximum) at different energies. This will ensure the consistency of the analysis in the fitting procedure.

XANES spectra were recorded at selected steps during the oxidation of a $1.2 \mathrm{~nm}$ thick $\mathrm{Fe}$ layer up to the saturation regime and subsequent deposition of Fe. The same procedure was applied to a $1.4 \mathrm{~nm}$ thick $\mathrm{Ni}$ layer. The evolution of the spectra during the oxidation is shown in Fig. 2. The onset of the absorption edge shifts toward higher energy with increasing oxygen exposure (measured in Langmuir, $1 \mathrm{~L}=1$ $\times 10^{-6}$ Torr s), indicating an overall increase in the oxidation state of the metal. The saturation regime is reached above $1300 \mathrm{~L}$ and only little changes are still observed at $6500 \mathrm{~L}$, indicating that the oxide growth stopped.

The deposition of metal on top of the saturated oxide affects the two systems in different ways. The corresponding 

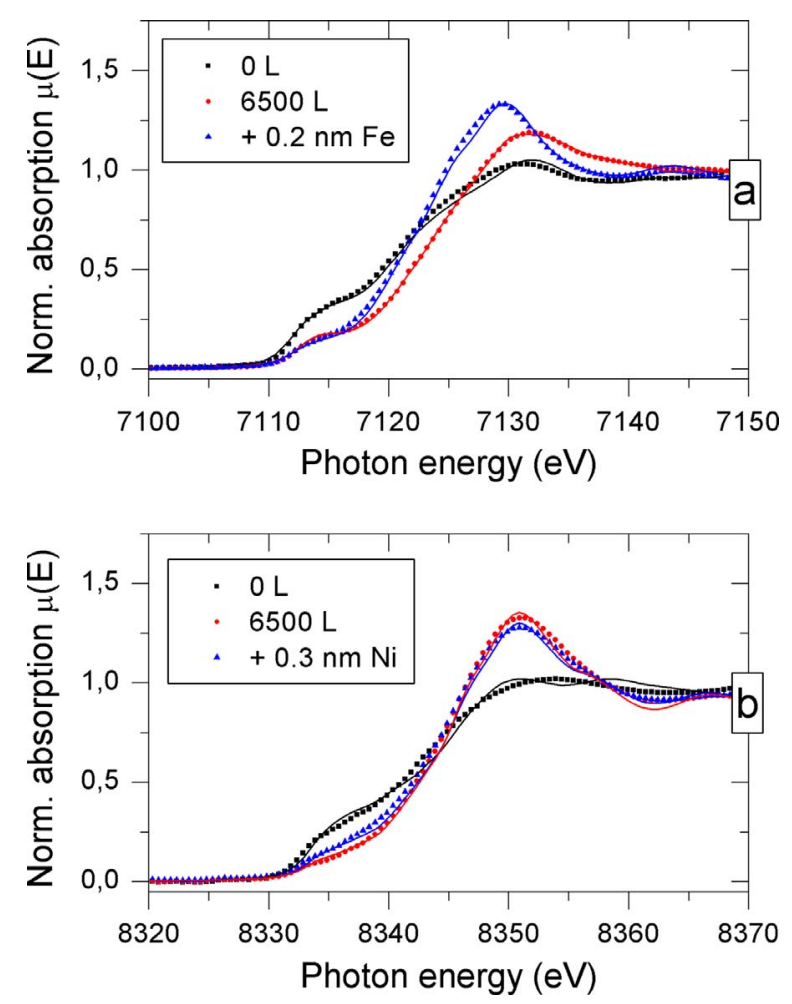

FIG. 3. (Color online) XANES $K$-edge spectra recorded at selected steps of the oxidation and subsequent deposition processes of (a) a $1.2 \mathrm{~nm}$ thick Fe layer and (b) a $1.4 \mathrm{~nm}$ thick Ni layer. The lines are fits using a linear combination of the reference spectra shown in Fig. 1.

XANES spectra for Fe and $\mathrm{Ni}$ are displayed in Figs. 3(a) and 3 (b), respectively. For the case of $\mathrm{Fe}$, the spectra change drastically and adopt a shape that was not encountered at any stage of the oxidation, whereas for the case of $\mathrm{Ni}$, only a slight decrease in the peak maximum is observed, without further changes in the spectra.

Quantitative values of the layer composition are obtained from the fitting procedure described earlier. The results are shown in Fig. 4. For the case of Fe, one sees that $\mathrm{Fe}^{2+}$ sites are created only at the early stage of the oxidation, whereas for $\mathrm{Fe}^{3+}$ (we omit the distinction between $\mathrm{Fe}_{\text {tet }}^{3+}$ and $\mathrm{Fe}_{\text {oct }}^{3+}$ ) the growth continues up to the saturation regime. This different growth mechanism is explained by a decreasing availability of Fe metal at the oxide/oxygen interface, which favors the growth of oxygen-rich $\mathrm{Fe}^{3+}$ type of sites. ${ }^{12,16}$ In saturation a mixture of $\mathrm{Fe}^{2+}$ and $\mathrm{Fe}^{3+}$ is present with apparently no direct stoichiometric relation to any of the bulk iron oxide phases. The case of nickel is somewhat different. It was possible to obtain good fits assuming a mixture of $\mathrm{Ni}$ metal $\left(\mathrm{Ni}^{0}\right)$ and $\mathrm{NiO}\left(\mathrm{Ni}^{2+}\right)$ only. This indicates that only $\mathrm{Ni}^{2+}$ sites are formed and that no oxygen-rich oxides are present in saturation. This behavior is not surprising as $\mathrm{NiO}$ is the most stable nickel oxide phase at room temperature. In fact the reduction potential of $\mathrm{Ni}^{3+}$ to $\mathrm{Ni}^{2+}$ is relatively large, ${ }^{17}$ and $\mathrm{Ni}^{3+}$ is therefore unlikely to form (or stay stable) under normal conditions. Indeed, $\mathrm{Ni}^{3+}$ is only seldomly observed in oxidation experiments. ${ }^{18,19,21}$ This behavior is in contrast to Fe, where the different oxidation states have similar oxidation poten-
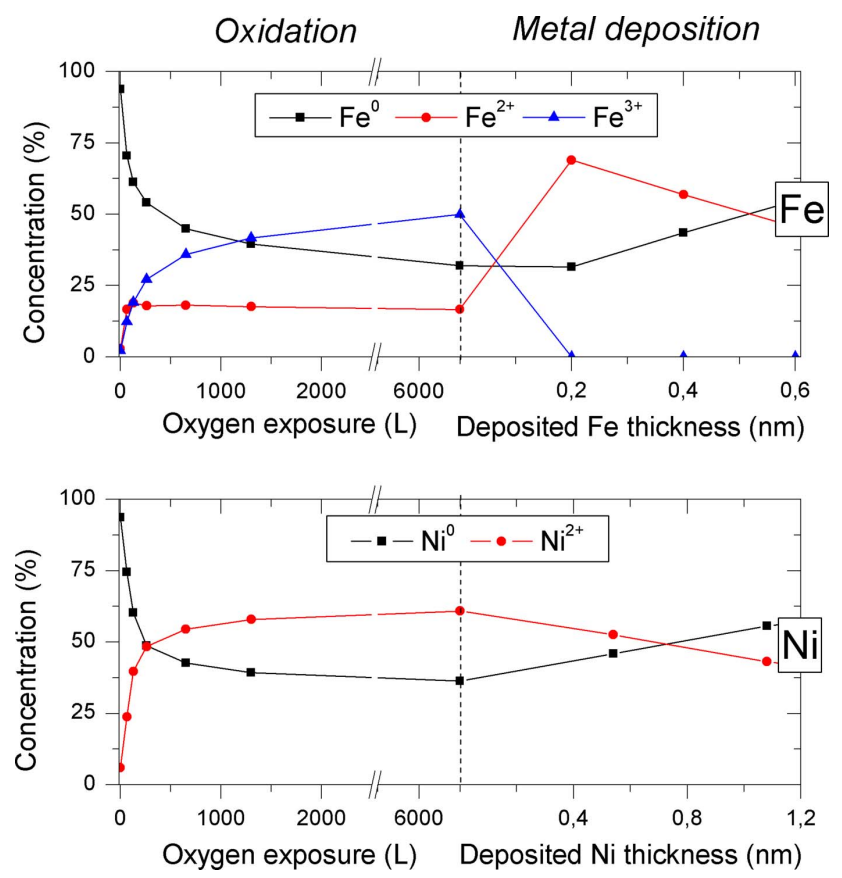

FIG. 4. (Color online) Evolution of the layer composition with increasing oxygen exposure (left) and deposited metal thickness (right), as derived from fitting of the XANES spectra shown in Figs. 2 and 3. Upper panel: Oxidation/Fe deposition on a nominally 1.2 $\mathrm{nm}$ thick Fe layer. Lower panel: Oxidation/Ni deposition of a nominally $1.4 \mathrm{~nm}$ thick Ni layer.

tials, and are therefore able to coexist at room temperature. One finally sees that the saturation regime is reached faster for the case of $\mathrm{Ni}$ oxidation, and that the total amount of oxygen atoms incorporated in the saturated oxide is $10 \%$ smaller compared to Fe.

Upon deposition of only $0.2 \mathrm{~nm} F e$ on the saturated $\mathrm{Fe}$ oxide, one sees that the drastic changes in the XANES spectra correspond to a complete reduction in the oxide layer to FeO. This particular effect has already been discussed in detail in Ref. 12 and involves strong interdiffusion processes at the newly created native-oxide/Fe interface, where it is expected that the metal adsorbate diffuses into the oxygen-rich oxide. For nickel, one observes only a change in the $\mathrm{Ni}^{0} / \mathrm{Ni}^{2+}$ ratio. This indicates that much less chemical interaction occurs between the $\mathrm{Ni}$ metal and its oxide compared to the case of $\mathrm{Fe}$.

These results are supported by analysis of the EXAFS part of the absorption signal, which is sensitive to the local order around the absorbing atom. Figure 5 shows the absolute values of Fourier transform (FT) modules of the EXAFS signal before and after deposition of metal on the saturated oxide for both metals. For Fe, strong changes in the radial distribution are seen. The small peak at $0.22 \mathrm{~nm}$ is originating from the underlying, not oxidized, metallic Fe. The increase in the second FeO shell peak is similar to what was reported in Ref. 12 and indicates that the deposition of metal has completely changed the oxide's structure. For $\mathrm{Ni}$, the peak shape does not change, supporting the interpretation that deposition of $\mathrm{Ni}$ does not lead to a change in the oxide chemistry. 


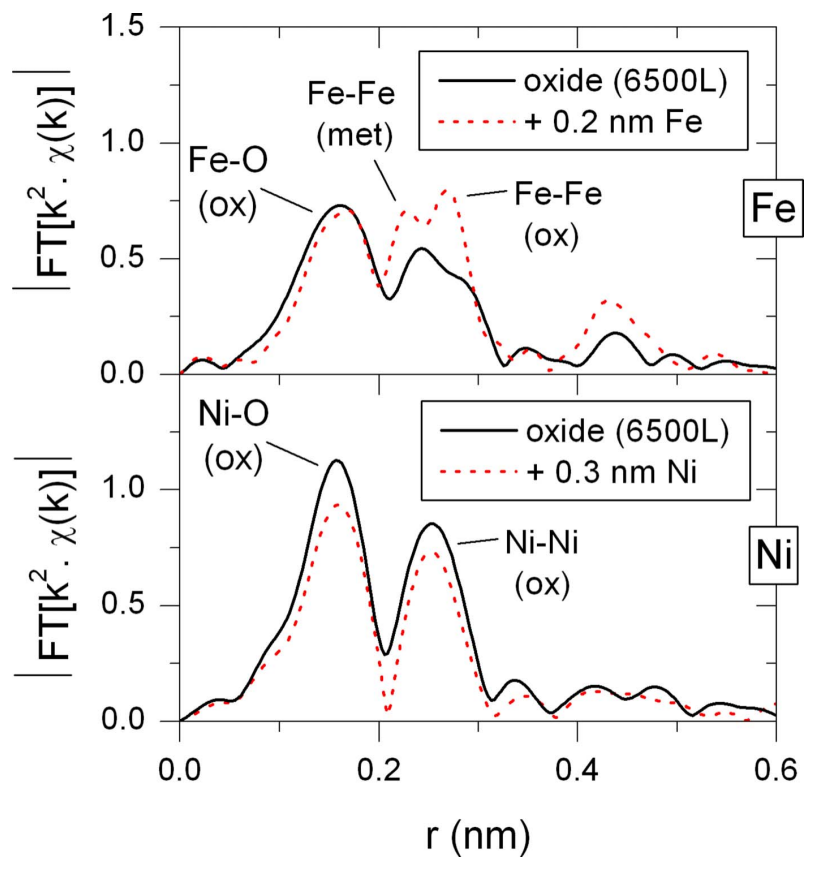

FIG. 5. (Color online) $k^{2}$ weighted Fourier transform (FT) modules of the EXAFS signal illustrating the change in local symmetry before and after deposition of metal on the saturated oxide (upper panel) for $\mathrm{Fe}$ and (lower panel) for $\mathrm{Ni}$.

One can conclude that the interface between the saturated metal oxide and its metal parent is formed under different conditions for the two metals. A reduction process occurs at the oxide/Fe interface, which leads to a diffusion of the metal into the oxide, whereas little interdiffusion is expected for $\mathrm{Ni}$, without further chemical changes.

\section{X-RAY REFLECTOMETRY}

The interface morphology, which is a result of the oxidation for the metal/oxide interface and of interdiffusion for the oxide/metal interface, was studied by $\mathrm{x}$-ray reflectometry. The technique is primarily sensitive to the electron-density depth profile. ${ }^{20}$ In the case of multilayer systems, this allows one to obtain the density and thickness of each layer, as well as the roughness of the different interfaces.

The x-ray reflectometry experiment was carried out at the W1 beamline of the DORIS III storage ring. The energy of the incoming $\mathrm{x}$-ray beam was monochromatized to $9.8 \mathrm{keV}$ using a $\mathrm{Si}(111)$ reflection. The reflectivity curves were recorded on superlattice samples with nominal structure $\mathrm{Si} /[\mathrm{Fe}(2.5 \mathrm{~nm}) / \mathrm{O}(6500 \mathrm{~L})]_{15} / \mathrm{Al}(3 \mathrm{~nm}) \quad$ and $\mathrm{Si} /[\mathrm{Ni}(3.1 \mathrm{~nm}) / \mathrm{O}(6500 \mathrm{~L})]_{13} / \mathrm{Pd}(3 \mathrm{~nm})$. The $6500 \mathrm{~L}$ exposure was chosen to reproduce the same oxidation conditions as in the XAS experiment. Only the sequence of deposition and oxidation was repeated several times to create superlattice samples. After oxidation, the subsequent metal deposition was started only after the background pressure fell below $2 \times 10^{-8}$ mbar.

The reflectivity curves for the $\mathrm{Fe}$ and $\mathrm{Ni}$ superlattices are displayed in the upper panel of Fig. 6. The fits were performed using a reduced set of parameters, assuming a repetition of the metal/oxide bilayers. The overall roughness was allowed to increase with an increasing number of layers. Fi-
$[\mathrm{Fe}(2.5 \mathrm{~nm}) / \mathrm{O}(6500 \mathrm{~L})]_{15}$
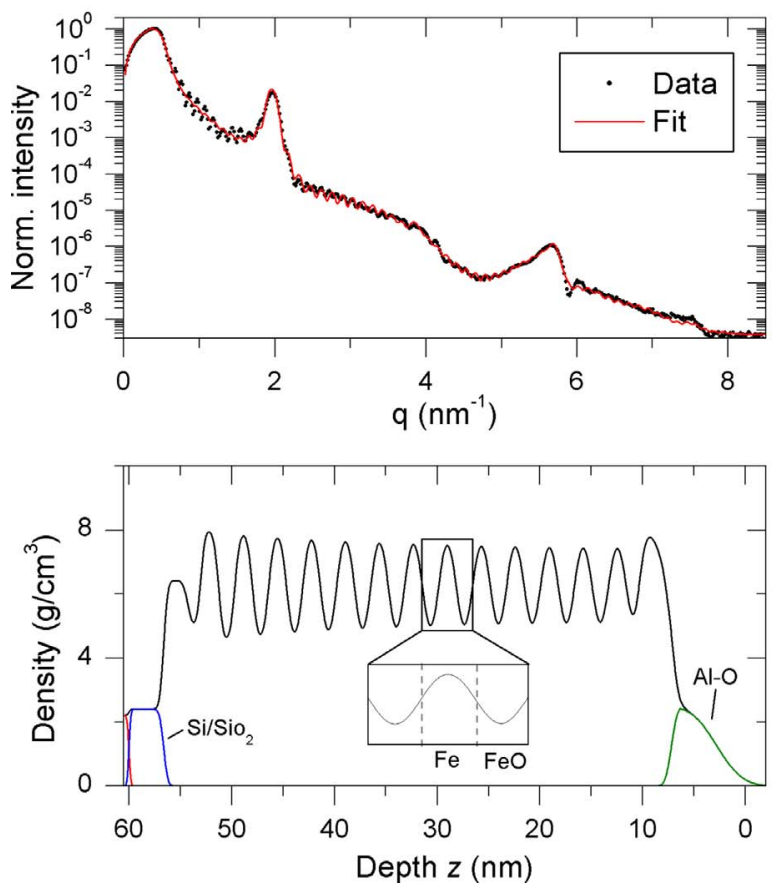

$[\mathrm{Ni}(3.1 \mathrm{~nm}) / \mathrm{O}(6500 \mathrm{~L})]_{13}$
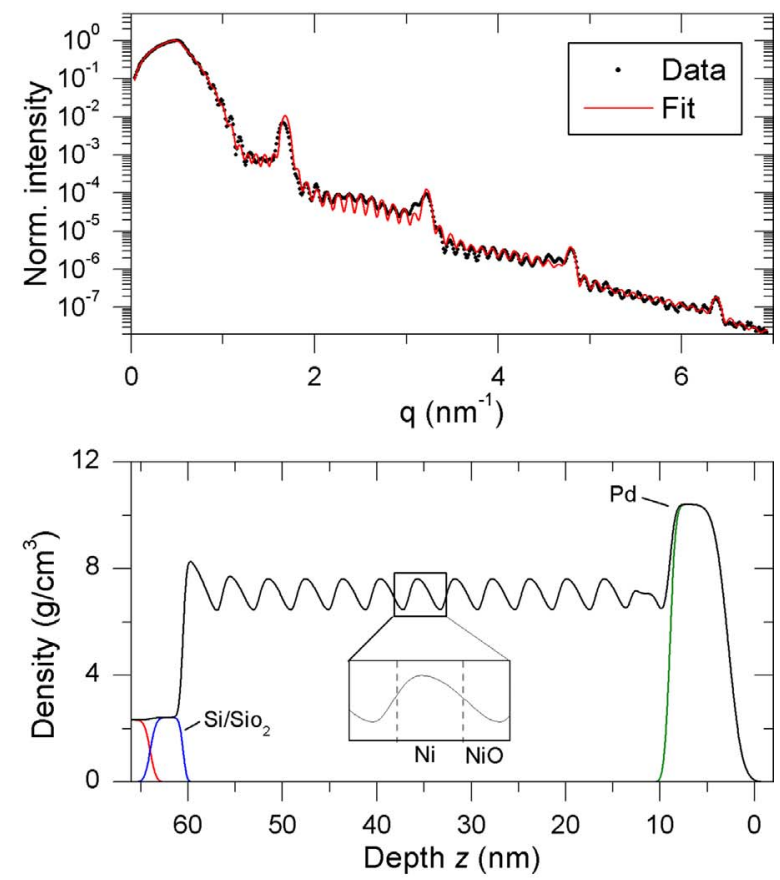

FIG. 6. (Color online) Upper panel: Reflectivity curve for (left) the Fe-based superlattice and (right) the nickel-based superlattice. The solid lines are fits. Lower panel: Depth density profile extracted from the simulation of the reflectivity curves. 
nally the density, thickness, and roughness of the lower bilayer (in contact with the $\mathrm{SiO}_{2}$ ) and the top bilayer (in contact with the capping layer) were varied. The suppression of the second-order Bragg peak for the Fe superlattice is a sensitive sign that both the metal and the oxide layers have almost equal thicknesses, which are found to be 1.69 and $1.61 \mathrm{~nm}$ for the metal and the oxide, respectively. The roughness is similar for the Fe/oxide and oxide/Fe interfaces and is found to increase progressively from $0.4 \mathrm{~nm}$ for the bottom layers to $0.7 \mathrm{~nm}$ for the top layers. For Ni, up to four Bragg peaks are seen in the reflectivity. The thicknesses extracted from the fit are of 2.46 and $1.49 \mathrm{~nm}$ for the $\mathrm{Ni}$ and $\mathrm{Ni}$-oxide layers, respectively. The measured root-mean-square (RMS) roughness is slightly increased compared to the Fe case and shows a strong asymmetry between the Ni/oxide and oxide/ $\mathrm{Ni}$ interfaces with values of 1.1 and $0.6 \mathrm{~nm}$, respectively.

It should be noted that the interface profile extracted from the fit is the only reasonable solution, which allows one to simultaneously fit the envelope of the curve and the four Bragg peaks at the same time. In fact, one observes that the absolute values of the roughness influence the envelope, while their relative values modify the profile of the four Bragg peaks. The good agreement between measured and calculated reflectivity rules out any other model of the interface roughness. In particular, it rules out the possibility for equivalent roughness at both interfaces or inversion of the asymmetry.

The reason for the increased roughness at the Ni/oxide interface compared to the Fe/oxide interface is not clear but is most probably related to the diffusion of ions involved in the oxidation procedure. In fact it is well known that the oxidation rate of polycrystalline $\mathrm{Ni}$ is enhanced due to grainboundary diffusion of the oxygen ${ }^{21}$ and that the oxidation usually proceeds via island formation. ${ }^{22}$ In principle, one can expect this nonhomogeneous oxide growth to be responsible for lateral heterogeneities in the oxide thickness, which possibly manifest as an increased roughness in the laterally integrated signal measured in a reflectivity experiment. For Fe oxidation, it is known that once a complete layer is formed, the oxidation can be described by a continuous layer model. $^{16}$

\section{DISCUSSION}

The symmetry (or asymmetry) of the metal/oxide and oxide/metal interfaces is presumably a result of the oxidation and reduction processes eventually occurring during the deposition of metal on the native oxide. For Ni, no reduction in the oxide occurs upon deposition of $\mathrm{Ni}$ on the $\mathrm{NiO}$. One can therefore assume that the subsequent metal growth simply follows the morphology of the underlying surface, with the consequence that the $\mathrm{NiO} / \mathrm{Ni}$ interface roughness is mainly determined by the oxidation process. In this case, the reduced interface roughness observed can eventually be attributed to a smoothening effect of the metal layer upon oxidation. For Fe, the oxidation can in principle also lead to a decrease in the surface roughness. However, the subsequent deposition of $\mathrm{Fe}$ metal leads to a reduction in the oxide which will modify the interface profile. In fact, one can view the $\mathrm{Fe}$ deposition on the oxide as a kind of oxidation procedure: Fe metal is deposited on an oxygen-rich surface and diffuses into the oxide, which in this case acts as a source of oxygen ions. From that perspective, a similar interface is formed at the oxide/metal interface as the one created during the oxidation process of the metal. The appearance of symmetric interfaces could therefore be a particular case which applies for Fe only. It should be noted that this study was performed by deposition of metal on its own oxide. This is in contrast to the study of Regan et al., ${ }^{8}$ which described the formation of TM/TMO interfaces where layers where constituted with different TM elements. Here, the use of the same TM for the metal and the oxide layers intrinsically rules out the influence of the different oxidation potentials attributed to different transition metals. The result shown here therefore highlights the influence of interdiffusion processes on the interface morphology. This should therefore be considered when growing metal on oxide substrates, even in the case where two different transition-metal elements are involved. In any case, one can expect that if reduction occurs, it will lead to an increase in the interface roughness.

Finally, the noncollinear magnetic coupling appearing between Fe layers separated by native-oxide spacer layers that was recently reported ${ }^{11}$ might be a consequence of the reduction process occurring upon deposition of metal on the oxide. In fact the magnetic coupling in these systems is expected to be due to interfacial exchange coupling between the ferromagnetic metal and the antiferromagnetic oxide. The noncollinear coupling is induced by the lateral variation in the oxide spacer thickness and is therefore dependent on the roughness of each interface. This, however, requires that both metal/oxide and oxide/metal interfaces have a similar morphology. As discussed in the previous paragraph, this could be a special case valid only for Fe/native-oxide multilayers. Further studies on magnetic coupling in other transition-metal/native-oxide systems would bring a deeper understanding of the interplay between interface structure and magnetism.

\section{CONCLUSIONS}

We have resolved the chemical changes occurring during the oxidation of $\mathrm{Fe}$ and $\mathrm{Ni}$ surfaces and the subsequent deposition of the same metal. It is observed that reduction in the oxygen-rich nonstoichiometric Fe oxide occurs upon deposition of Fe. This process leads to the appearance of symmetric $\mathrm{Fe} /$ oxide and oxide/Fe interfaces. The case of $\mathrm{Ni}$, where no reduction occurs, shows a strong asymmetry in the interface morphology. These results highlight the reduction process occurring at oxide/transition-metal interfaces as the main cause of symmetric interfaces in Fe/native-oxide multilayers. The differing oxidation and reduction processes for $\mathrm{Fe}$ and Ni mainly arise because at room temperature several oxidation states (and crystallographic sites) can coexist for $\mathrm{Fe}$ and not for Ni. Thus, understanding the interaction of Fe metal with its different oxide phases is of utmost importance for layer systems incorporating these oxides.

This study combines x-ray spectroscopy and reflectometry to investigate the role of chemical processes on the structure 
during the formation of an interface. The use of highresolution x-ray reflectometry proved to be a decisive tool to study the interface density profile with high resolution. The technique used here can be applied to a wide range of systems (in particular for the interface formation of reactive compounds). In that view, performing such experiments on a synchrotron beamline optimized for both absorption and reflectometry measurements would allow one to observe chemical and structural changes at every step of the growth.

\section{ACKNOWLEDGMENT}

The authors would like to thank E. Welter and D. Zajac from HASYLAB for their help during the x-ray absorption experiment. W. Caliebe and O. Seeck are greatly acknowledged for the support provided at the W1 beamline. K. S. is indebted to the Slovak Grant Agency for Science for financial support (Grant No. 2/7196/27).
*Author to whom correspondence should be addressed: ralf.roehlsberger@desy.de

${ }^{1}$ S. Parkin, X. Jiang, C. Kaiser, A. Panchula, K. Roche, and M. Samant, Proc. IEEE 91, 661 (2003).

${ }^{2}$ Y. Ijiri, J. Phys.: Condens. Matter 14, R947 (2002).

${ }^{3}$ M. Finazzi, L. Duó, and F. Ciccacci, Surf. Sci. Rep. 62, 337 (2007).

${ }^{4}$ A. E. Berkowitz and K. Takano, J. Magn. Magn. Mater. 200, 552 (1999).

${ }^{5}$ G. Nowak, A. Remhof, F. Radu, A. Nefedov, H.-W. Becker, and H. Zabel, Phys. Rev. B 75, 174405 (2007).

${ }^{6}$ H. Ohldag, T. J. Regan, J. Stöhr, A. Scholl, F. Nolting, J. Lüning, C. Stamm, S. Anders, and R. L. White, Phys. Rev. Lett. 87, 247201 (2001).

${ }^{7}$ L. Q. Pan, G. M. Zhang, C. F. Fan, H. Qiu, P. Wu, F. P. Wang, and Y. Zhang, Thin Solid Films 473, 63 (2005).

${ }^{8}$ T. J. Regan, H. Ohldag, C. Stamm, F. Nolting, J. Lüning, J. Stöhr, and R. L. White, Phys. Rev. B 64, 214422 (2001).

${ }^{9}$ M. Gruyters, J. Magn. Magn. Mater. 248, 248 (2002).

${ }^{10}$ G. S. D. Beach and A. E. Berkowitz, IEEE Trans. Magn. 41, 2053 (2005).

${ }^{11}$ Th. Diederich, S. Couet, and R. Röhlsberger, Phys. Rev. B 76,
054401 (2007).

${ }^{12}$ S. Couet, K. Schlage, K. Saksl, and R. Röhlsberger, Phys. Rev. Lett. 101, 056101 (2008).

${ }^{13} \mathrm{~S}$. Couet, Th. Diederich, K. Schlage, and R. Röhlsberger, Rev. Sci. Instrum. 79, 093908 (2008).

${ }^{14}$ A. Erbil, G. S. Cargill, R. Frahm, and R. F. Boehme, Phys. Rev. B 37, 2450 (1988).

${ }^{15}$ D. V. Dimitrov, K. Unruh, G. C. Hadjipanayis, V. Papaefthymiou, and A. Simopoulos, Phys. Rev. B 59, 14499 (1999).

${ }^{16}$ S. J. Roosendaal, A. M. Vredenberg, and F. H. P. M. Habraken, Phys. Rev. Lett. 84, 3366 (2000).

${ }^{17}$ S. G. Bratsch, J. Phys. Chem. Ref. Data 18, 1 (1989).

${ }^{18}$ P. H. Holloway and J. B. Hudson, Surf. Sci. 43, 123 (1974).

${ }^{19}$ K. Yagi-Watanabe, Y. Ikeda, Y. Ishii, T. Inokuchi, and H. Fukutani, Surf. Sci. 482-485, 128 (2001).

${ }^{20}$ M. Tolan, X-ray Scattering from Soft-Matter Thin Films, Springer Tracts in Modern Physics (Springer-Verlag, Berlin, 1999), Vol. 148.

${ }^{21}$ B. P. Payne, A. P. Grosvenor, M. C. Biesinger, B. A. Kobe, and N. S. McIntyre, Surf. Interface Anal. 39, 582 (2007).

${ }^{22}$ E. Kopatzki and R. J. Behm, Phys. Rev. Lett. 74, 1399 (1995). 